\title{
Cancellation and stability properties of generalized torsion modules
}

\author{
F.E.A. Johnson
}

\begin{abstract}
Given a module $X$ over a ring $\Lambda$ its stability class consists of all modules $X^{\prime}$ such that $X \oplus \Lambda^{a} \cong X^{\prime} \oplus \Lambda^{b}$ for some positive integers $a, b$. If the ring $\Lambda$ is weakly finite then the stability class of a finitely generated $\Lambda$-module has the structure of a tree. We show that if, in addition, $X$ is a generalized torsion module its stability class has the same shape as that of the zero module. In consequence we construct examples of non-projective modules whose stability classes have arbitrarily large amounts of branching.
\end{abstract}

Keywords: Cancellation, stable module, generalized torsion module.

Mathematics Subject Classification (AMS 2010): 16D70; 16S90; $20 E 06$.

Let $\Lambda$ be a ring and let $N$ be a $\Lambda$-module; the cancellation problem for $N$ asks whether, for arbitrary $\Lambda$-modules $M, M^{\prime}$, the existence of an isomorphism $M^{\prime} \oplus N \cong M \oplus N$ implies that $M^{\prime} \cong M$. In practice, we restrict attention to modules which are finitely generated. In this degree of generality, there is a considerable literature to show that one expects the answer to be 'no' even over rings $\Lambda$ which in other respects are comparatively well behaved (cf [11], [18]). Given this general failure, the particular case when $N=\Lambda$ nevertheless remains of considerable interest; thus we ask:

(†) If $M^{\prime} \oplus \Lambda \cong M \oplus \Lambda$ what further conditions on $M$ imply that $M^{\prime} \cong M$ ?

More generally we consider the stable class $[M]$ of $M$ which consists of all modules $M^{\prime}$ such that $M^{\prime} \oplus \Lambda^{a} \cong M \oplus \Lambda^{b}$ for some positive integers $a, b$. If $M$ is finitely generated then, as we shall recall in $\S 1$, under a mild restriction, weak finiteness (cf [4], [5]), on the ring $\Lambda,[M]$ has the structure of a directed tree. A more general problem than $(\dagger)$ is

(††) If $M$ is a finitely generated $\Lambda$-module, describe the tree structure on $[M]$.

In the case of modules over the integral group rings of finite groups there is an extensive literature on the tree structures of quite general modules (cf. [6], [9], [10], [15], [17]). By contrast, far less is known about the stable classes of modules over infinite groups. Moreover, such results as are known are confined almost exclusively 
to projective modules (although see [7] for the special case of the augmentation ideal). In this paper we are concerned with modules which are far from being projective.

The starting point in any such study is to describe the tree structure on [0], the stable class of the zero module, the elements of which are the isomorphism classes of (finitely generated) stably free $\Lambda$-modules. This example is in some sense universal as there is a mapping of trees $\nu:[0] \rightarrow[M]$ defined by $\nu(S)=M \oplus S$. We say that $M$ is a generalized torsion module when $\operatorname{Hom}_{\Lambda}(M, \Lambda)=0$; we shall prove:

If $M$ is a finitely generated generalized torsion module then $\nu:[0] \rightarrow[M]$ is an isomorphism of trees.

In particular, when $\nu$ is a tree isomorphism it necessarily preserves height; $M$ is then the unique module at the minimal level of its stable class and one gets a definite solution to $(\dagger)$ as follows:

(II) If $M$ is a generalized torsion module and $M^{\prime} \oplus \Lambda \cong M \oplus \Lambda$ then $M^{\prime} \cong M$.

In $\S 1$ we give a brief account of stable modules over weakly finite rings. (I) and (II) are proved in $\S 2$. In $\S 3$ we give examples to which these statements then apply. In particular, we construct examples of generalized torsion modules whose trees have arbitrary amounts of branching at level 1. Our construction relies on the existence of nontrivial stably free modules known from other sources. We wish to thank the referee for a number of helpful and clarifying suggestions.

\section{$\S 1$ : Stable modules and tree structures :}

We say the ring $\Lambda$ has the weak finiteness property (cf [5]) when, for any integer $a \geq 1$

(WF) If $\varphi: \Lambda^{a} \rightarrow \Lambda^{a}$ is a surjective $\Lambda$-homomorphism then $\varphi$ is bijective.

We note that :

(1.1) If $R$ is a commutative integral domain of characteristic zero then, for any group $G$, the group ring $R[G]$ is weakly finite.

According to Cohn [4], (1.1) was first stated by Kaplansky although it seems he never published a proof. However a proof was eventually published in a paper of Montgomery [12] although there the term 'stably finite' is used instead of 'weakly finite'.

For the rest of this paper we will assume, without further mention, that $\Lambda$ is a weakly finite ring. We note a number of properties that finitely generated $\Lambda$-modules thereby possess, the details of which can be found in Chapter 1 of [8]. Firstly, we denote by ' $\sim$ ' the stability relation on $\Lambda$ modules; that is

$$
M_{1} \sim M_{2} \Longleftrightarrow M_{1} \oplus \Lambda^{a} \cong M_{2} \oplus \Lambda^{b}
$$


for some integers $a, b \geq 0$; the relation ' $\sim$ ' is an equivalence on isomorphism classes of $\Lambda$-modules. For any $\Lambda$-module $M$, we denote by $[M]$ the corresponding stable module; that is, the set of isomorphism classes of modules $N$ such that $N \sim M$. If $M$ is a finitely generated $\Lambda$-module there is a well defined function $g:[M] \times[M] \rightarrow \mathbb{Z}$, the 'gap function' given as follows

$$
g\left(N_{1}, N_{2}\right)=g \quad \Longleftrightarrow N_{1} \oplus \Lambda^{a+g} \cong N_{2} \oplus \Lambda^{a}
$$

where both $a$ and $a+g$ are positive integers. We say that a module $M_{0} \in[M]$ is a root module for $[M]$ when $0 \leq g\left(M_{0}, N\right)$ for all $N \in[M]$. We note the following ([8] p.6) :

(1.2) If $M$ is a finitely generated $\Lambda$-module then $[M]$ contains a root module.

If $M_{0}$ is a root module for $[M]$ we may define a height function $h:[M] \rightarrow \mathbb{N}$ by

$$
h(N)=g\left(M_{0}, N\right) .
$$

Evidently root modules are precisely those of minimal height; that is:

(1.3) If $M^{\prime} \in[M]$ then $M^{\prime}$ is a root module precisely when $h\left(M^{\prime}\right)=0$.

When $M$ is a finitely generated $\Lambda$-module, the stable module $[M]$ has the structure of a graph in which the vertices are the isomorphism classes of modules $N \in[M]$ and where we draw an edge $N_{1} \rightarrow N_{2}$ when $N_{2} \cong N_{1} \oplus \Lambda$.

(1.4) If $M$ is a finitely generated $\Lambda$-module then $[M]$ is an infinite (directed) tree.

The existence of the height function $h:[M] \rightarrow \mathbb{N}$ implies that $[M]$ may be represented as a infinite tree whose roots do not extend infinitely downwards. Determining the precise shape of the tree $[M]$ is equivalent to finding all modules (?) which solve the equation

$$
(?) \oplus \Lambda^{a} \cong M \oplus \Lambda^{b}
$$

for some integers $a, b$. In particular, the shape of the stable class [0] of the zero module is of fundamental importance. Recall that a $\Lambda$-module $S$ is said to be stably free when $S \oplus \Lambda^{a} \cong \Lambda^{b}$; so solving (1.5) in the case $M=0$; that is, the stably free modules constitute the stable class [0].

It is instructive to give some examples. The diagram below illustrates the notion, with (A) showing proper branching and (B) representing the trivial case where there is no branching at all:

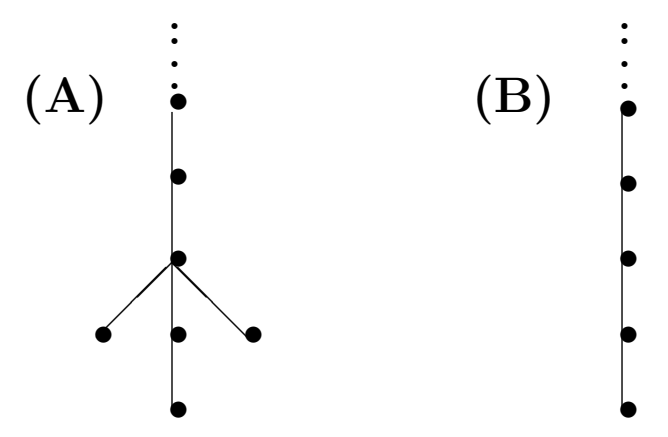


In fact both trees actually arise from stable modules; according to the calculations of Swan [17], (A) represents the stable class [0] of the zero module over the integral group ring $\mathbb{Z}[Q(24)]$ of the quaternionic group of order 24 whereas, in the light of the Quillen-Suslin solution [13], [14] to the Serre conjecture, (B) represents the stable class [0] of the zero module over the integral group ring $\mathbb{Z}\left[C_{\infty}^{n}\right]$ of the free abelian group of rank $n$; see also [16].

Stable modules with the shape of (B) are said to be straight; formally, the stable module $[M]$ is straight when up to isomorphism there is a unique module $M_{0}$ at height 0 and such that, if $N \in[M]$ then $N \cong M_{0} \oplus \Lambda^{n}$ for some integer $n \geq 0$. We say that $\Lambda$ has stably free cancellation $(=S F C)$ when the stable class [0] is straight.

\section{$\S 2$ : The stable class of a generalized torsion module:}

In this section $\Lambda$ will denote a weakly finite ring. Let $M_{1}, M_{2}, N_{1}, N_{2}$ be modules over $\Lambda$ and suppose that $\Phi: M_{1} \oplus M_{2} \rightarrow N_{1} \oplus N_{2}$ is a $\Lambda$-isomorphism. We may describe $\Phi$ as a $2 \times 2$ matrix of $\Lambda$-homomorphisms $\Phi=\left(\varphi_{i j}\right)_{1 \leq i, j \leq 2}$ where $\varphi_{i j}: M_{j} \rightarrow N_{i}$. We note:

(2.1) If $\Phi$ is an isomorphism and $\varphi_{21}=0$ then $\varphi_{11}$ is injective and $\varphi_{22}$ is surjective.

A module $M$ is a generalized torsion module when $\operatorname{Hom}_{\Lambda}(M, \Lambda)=0$; equivalently when $\operatorname{Hom}_{\Lambda}(M, P)=0$ for any projective $\Lambda$-module $P$.

Theorem 2.2 : $\quad$ Let $M, M^{\prime}$ be $\Lambda$ modules such that $M \oplus S \cong M^{\prime} \oplus S^{\prime}$ where $S$, $S^{\prime}$ are stably free $\Lambda$-modules of ranks $m, m^{\prime}$ respectively. If $M$ is a generalized torsion module then

i) $\quad m^{\prime} \leq m$ and

ii) $M^{\prime} \cong M \oplus T$ where $T$ is a stably free $\Lambda$-module of rank $m-m^{\prime}$.

Proof : Suppose that $\Phi: M \oplus S \rightarrow M^{\prime} \oplus S^{\prime}$ is an isomorphism and represent $\Phi$ as a matrix

$$
\Phi=\left(\begin{array}{cc}
\varphi_{11}: M \rightarrow M^{\prime} & \varphi_{12}: S \rightarrow M^{\prime} \\
\varphi_{21}: M \rightarrow S^{\prime} & \varphi_{22}: S \rightarrow S^{\prime}
\end{array}\right) .
$$

As $M$ is generalized torsion then $\varphi_{21}=0$. Hence, by (2.1), $\varphi_{22}: S \rightarrow S^{\prime}$ is surjective. As $\Lambda$ is weakly finite it follows that $m^{\prime} \leq m$. Moreover, also by $(2.1), \varphi_{11}: M \rightarrow M^{\prime}$ is injective. Put $N=\operatorname{Im}\left(\varphi_{11}\right) \subset M^{\prime}$. As $\varphi_{21}=0$ then $\Phi$ induces an isomorphism

$$
\Phi_{*}:(M \oplus S) / M \stackrel{\simeq}{\longrightarrow}\left(M^{\prime} \oplus S^{\prime}\right) / N .
$$

However $(M \oplus S) / M \cong S$ and, as $N \subset M^{\prime}$ then $\left(M^{\prime} \oplus S^{\prime}\right) / N \cong\left(M^{\prime} / N\right) \oplus S^{\prime}$; hence

$$
\left(M^{\prime} / N\right) \oplus S^{\prime} \cong S
$$


Put $T=M^{\prime} / N$. Then $T$ is stably free and, as $\Lambda$ is weakly finite, its rank is well defined, namely $m-m^{\prime}$. We now have an exact sequence $0 \rightarrow N \rightarrow M^{\prime} \rightarrow T \rightarrow 0$ which splits as $T$ is projective. Thus $M^{\prime} \cong N \oplus T$ from which ii) follows as $\varphi_{11}$ maps $M$ isomorphically onto $N$.

From (2.2) it follows directly that:

Corollary 2.3 : If $M$ is a generalized torsion module then, up to isomorphism, $M$ is the unique representative at the minimal level of the stability class $[M]$.

Observe that (2.3) implies statement (II) of the Introduction. A slight variation on the proof of (2.2) shows that:

Proposition 2.4 : Let $M$ be a generalized torsion module and let $S, S^{\prime}$ be stably free $\Lambda$-modules of the same rank $n$; then $M \oplus S^{\prime} \cong M \oplus S$ if and only if $S^{\prime} \cong S$.

Proof : Proceed as in the proof of (2.2) but with $M^{\prime}=M$. Then $(M / N) \oplus S^{\prime} \cong S$ so that $M / N$ is a stably free module of $\operatorname{rank} \operatorname{rk}(S)-\operatorname{rkS} S^{\prime}=0$. Hence $M / N=0$ and $S^{\prime} \cong S$. The converse is trivial.

The stability class [0] consists of all finitely generated stably free $\Lambda$-modules. For any finitely generated $\Lambda$-module $M$ there is a mapping of directed trees $\nu:[0] \rightarrow[M]$ given by

$$
\nu(S)=M \oplus S .
$$

From (2.2), (2.3) and (2.4) we now deduce statement (I) of the Introduction, namely:

Corollary 2.5 : If $M$ is a finitely generated generalized torsion module then $\nu:[0] \rightarrow[M]$ is an isomorphism of directed trees.

Corollary 2.6 : If $M$ is a finitely generated generalized torsion module the stability class $[M]$ is straight if and only if $\Lambda$ has property $S F C$.

\section{$\S 3$ : Virtually trivial modules :}

Throughout this section $G$ will denote a group and $\Lambda$ will denote the group ring $\Lambda=R[G]$ where $R$ is a commutative integral domain of characteristic zero; moreover, $K$ will denote a subgroup of $G$ whose meaning is fixed throughout. If $M$ is a $\Lambda$-module and $m \in M$ we denote by $\langle m\rangle$ the orbit of $m$ under $K$

$$
\langle m\rangle=\{m \cdot k \mid k \in K\} .
$$

We say that $M$ is virtually trivial (resp. trivial) with respect to $K$ when for each $m \in M$ the orbit $\langle m\rangle$ is finite (resp. a singleton). 
Proposition 3.1: If $K$ is infinite and $M$ is virtually trivial with respect to $K$ then $M$ is a generalized torsion module.

Proof : As a module over $R[K], \Lambda$ is free of rank $|G / K|$. Let $\left(\mathbf{e}_{t}\right)_{t \in G / K}$ be an $R[K]$ basis for $\Lambda$. Then for each $\alpha \in \Lambda$ there is a matrix $\left(a_{k}^{t}\right)$ indexed by $t \in G / K$ and $k \in K$ with entries in $R$, only finitely many of which are nonzero, such that

$$
\alpha=\sum_{t \in G / K} \sum_{k \in K} \mathbf{e}_{t} a_{k}^{t} \cdot k=\sum_{k \in K} \alpha_{k} \cdot k
$$

where $\alpha_{k}=\sum_{t \in G / K} \mathbf{e}_{t} a_{k}^{t}$. We define $\operatorname{supp}(\alpha)=\left\{k \in K \mid \alpha_{k} \neq 0\right\}$. Under the assumption that $M$ is virtually trivial let $f: M \rightarrow \Lambda$ be a $\Lambda$-linear mapping and let $m \in M$. Put $S=\operatorname{supp}(f(m))$. We claim that

(*) $S=\emptyset$.

Observe that, for all $\gamma \in K, \operatorname{supp}(f(m) \cdot \gamma)=S \cdot \gamma$. Now write $\langle m\rangle=\left\{m_{1}, \ldots, m_{n}\right\}$ where $m=m_{1}$. For each $r$, choose $k_{r} \in K$ such that $m_{r}=m \cdot k_{r}$ and put $S(r)=S \cdot k_{r}$. Then $\mathfrak{S}=\bigcup_{r=1}^{n} S(r)$ is finite. As $K$ is infinite we may choose $\gamma \in K$ such that $\mathfrak{S} \cdot \gamma \bigcap \mathfrak{S}=\emptyset$. As $S=S(1)$ then $S \cdot \gamma \bigcap \mathfrak{S}=\emptyset$. However $f(m) \cdot \gamma=f(m \cdot \gamma)$ and as $m \cdot \gamma \in\langle m\rangle$ then, for some $r, m \cdot \gamma=m \cdot k_{r}$ so that $f(m) \cdot \gamma=f(m) \cdot k_{r}$ and $S \cdot \gamma=S(r)$. Thus $S \cdot \gamma \subset \mathfrak{S}$ and $S \cdot \gamma \bigcap S \cdot \gamma=\emptyset$. Thus $S \cdot \gamma=\emptyset$ so that $S=\emptyset$ as claimed. Hence $f(m)=0$. As this is true for all $m$ then $f=0$.

Suppose $1 \rightarrow K \stackrel{i}{\rightarrow} G \stackrel{\pi}{\rightarrow} H \rightarrow 1$ is an exact sequence of groups and put $\Lambda=\mathbb{Z}[G]$. If $M$ is a module over $\mathbb{Z}[H]$ we denote by $\widehat{M}=\pi^{*}(M)$ the $\Lambda$-module coinduced from $M$; that is, the underlying additive group of $\widehat{M}$ is that of $M$ and the $G$ action ' $\diamond$ ' is given by

$$
m \diamond g=m \cdot \pi(g) .
$$

Evidently $\widehat{M}$ is trivial with respect to $K$ so that, if $K$ is infinite then by Theorem I and (3.1), it follows that $\nu:[0] \stackrel{\simeq}{\longrightarrow}[\widehat{M}]$ is an isomorphism of directed trees. At this level of generality, however, one loses control over the structure of the stable class [0]. One can re-assert control by restricting the nature of $G$ as we proceed to show.

Let $\Gamma, H$ be nontrivial groups and denote by $G$ the free product $G=\Gamma * H$. Let $i_{\Gamma}: \Gamma \hookrightarrow G$ be the canonical inclusion and $\pi_{\Gamma}: G \rightarrow \Gamma, \pi_{H}: G \rightarrow H$ the canonical projections. Put $K=\operatorname{Ker}\left(\pi_{H}\right)$. Then $K$ is infinite by the Kurosh Theorem. Now take $M$ to be a finitely generated, but otherwise arbitrary, module over $\mathbb{Z}[H]$; then $\widehat{M}$ is trivial with respect to $K$ and so, by $(2.5)$ and $(3.1),[\widehat{M}]$ has same amount of branching as the stable class $[0]$ over $\mathbb{Z}[G]$. As a first example we note:

(i) If $\Gamma$ is a free group and $H=C_{\infty}$ then $[\widehat{M}]$ is straight ([2]). 
For the sake of definiteness we continue to take $H$ to be the infinite cyclic group $H=C_{\infty}$. To construct examples where $[\widehat{M}]$ has branching we now construct free products $G=\Gamma * C_{\infty}$ for which $\Lambda=\mathbb{Z}[G]$ admits nontrivial stably free modules. Observe that if $S$ is a stably free module over $\Gamma$ then the induced module $T=\left(i_{\Gamma}\right)_{*}(S)$ is a stably free module over $\Lambda$. Moreover, as $\pi_{\Gamma} \circ i_{\Gamma}=\operatorname{Id}_{\Gamma}$ then $\left(\pi_{\Gamma}\right)_{*}(T) \cong\left(\pi_{\Gamma} \circ i_{\Gamma}\right)_{*}(S) \cong S$. Consequently, if $S$ is nonfree then $T$ is also nonfree. Thus by varying $\Gamma$ we may construct groups $G$ in which the stable class of [0], and thereby also of $[\widehat{M}]$, has arbitrarily large amounts of branching. First consider the quaternionic group $Q(4 n)$ of order $4 n$ :

$$
Q(4 n)=\left\langle x, y \mid x^{n}=y^{2}, y x y^{-1}=x^{-1}\right\rangle .
$$

(ii) When $\Gamma=Q(4 n)$ then, by the calculations of Swan [17], the amount of branching at level 1 in $[\widehat{M}]$ increases as $n$ increases.

(iii) If $\Gamma=C_{\infty} \times Q(8 n)$ then, by the results of [8] Chap 12, [ $\left.\widehat{M}\right]$ has an infinite amount of branching at level 1 .

(iv) Take $\Gamma$ to be the poly\{infinite cyclic\} group constructed by Berridge and Dunwoody in [3]. Then the stable class $[0]$, hence also $[\widehat{M}]$, has non-trivial branching. In this case $G$ has cohomological dimension three and hence is torsion free.

(v) A similar conclusion to (iv) follows if we take $\Gamma$ to be the direct product of a free abelian group of rank $m$ with the fundamental group of the Klein bottle; it follows by a result of Artamonov ([1]) that the stable class [0], hence also $[\widehat{M}]$, has non-trivial branching. In this case $G$ is again torsion free and has cohomological dimension $m+2$.

\section{F.E.A. Johnson}

Department of Mathematics

University College London

Gower Street, London WC1E 6BT, U.K.

e-mail address : feaj@math.ucl.ac.uk 


\section{REFERENCES}

[1] : V.A. Artamonov ; Quantum Serre's problem : (in Russian) Uspekhi. Math. Nauk. 53 (1998) 3-77.

[2] : H. Bass ; Projective modules over free groups are free : J. of Algebra 1 (1964) 367-373.

[3] : P.H. Berridge and M. Dunwoody ; Non-free projective modules for torsion free groups: J. London Math. Soc. (2) 19 (1979) 433-436.

[4] : P.M. Cohn ; Some remarks on the invariant basis property : Topology 5 (1966) 215-228.

[5] : P.M. Cohn : Skew fields : Theory of general division rings. CUP (1995)

[6] : H. Jacobinski ; Genera and decompositions of lattices over orders. Acta Math. 121 (1968) 1-29.

[7] : F.E.A. Johnson ; The stable class of the augmentation ideal : K-Theory 34 (2005) 141-150.

[8] : F.E.A. Johnson; Syzygies and homotopy theory. Springer Verlag, 2011.

[9] : F.E.A. Johnson; A cancellation theorem for generalized Swan modules. Illinois Journal of Mathematics. 63 (2019) 103-125.

[10] : F.E.A. Johnson; Metacyclic groups and the D(2)-problem. World Scientific, 2021.

[11] : L.Klingler; Modules over the integral group ring of a non-abelian group of order $p q$. Memoirs A.M.S, no. 341 (1986).

[12] : S. Montgomery; von Neumann finiteness of tensor products of algebras. Communications in Algebra 11 (1983) 595-610.

[13] : D.G. Quillen; Projective modules over polynomial rings. Inventiones Math. 36 (1976) 167-171.

[14]: A.A. Suslin; Projective modules over a polynomial ring are free. Soviet Math. Dokl. 17 (1976) 1160-1164.

[15] : R.G. Swan ; K-Theory of finite groups and orders. (notes by E.G. Evans ). Lecture Notes in Mathematics 149. Springer-Verlag 1970.

[16] : R.G. Swan ; Projective modules over Laurent polynomial rings. Trans. Amer. Math. Soc. 237 (1978) 111-120.

[17] : R.G. Swan ; Projective modules over binary polyhedral groups. Journal für die Reine und Angewandte Mathematik. 342 (1983) 66-172.

[18] : R.G. Swan; Torsion-free cancellation over orders. Illinois Journal of Mathematics 32 (1988), 329-360. 